\title{
Der Mistlauer Taufstreit 1747 bis 1754
}

\author{
vON ULRICH FrÖHNER
}

Mistlau ist ein kleines Dorf in Hohenlohe. Heute gehört es zur Stadt Kirchberg/ Jagst und zählt rund 75 Einwohner. Es war im 18. Jahrhundert zur Zeit der Gegenreformation Schauplatz eines Machtkampfes zwischen der evangelischen Grafschaft Hohenlohe und dem katholischen Stift Comburg bei Schwäbisch Hall. Der Streit ist in den Archiven von Ludwigsburg und Neuenstein gut dokumentiert und liefert ein anschauliches Bild von den damaligen kirchlichen und herrschaftlichen Verhältnissen. Der Streit dauert insgesamt von 1681 bis zum Ende der Hohenloher Herrschaft im Jahr 1807. Besonders heftig tobte er in den Jahren 1747 bis 1754 - auf diesen Zeitraum konzentriert sich deshalb die folgende Darstellung.

\section{Der Pfarrer von Gaggstatt erhält einen Brief}

Damals wie heute war Mistlau eine Filiale des evangelische Pfarramts im benachbarten Gaggstatt (heute ebenfalls Gemeinde Kirchberg/Jagst). Der evangelische Pfarrer dort erhielt am 11. Februar 1747 den folgenden Brief ${ }^{1}$ :

Weilen auf das von hier in besagter Mistlauer Angelegenheit an das Stift Comburg schon vor einiger Zeit ergangene Vorstellungs-Schreiben, die endlich ohnlängst eingelangte Antwort so nicht beschaffen ist, umb sich zu einem gütlichen Austrag dermahlen und in Kürze Hoffnung machen zu können, [.. .].

Es gab also ein Beschwerde-Schreiben von Kirchberg an das Stift Comburg mit einer unbefriedigenden Antwort. Und offenbar war keine gütliche Einigung in Sicht. Im Brief geht es dann weiter: Weil Michel Bergers Weib hingegen Ihrer Geburts-Zeit nahe seyn wolle; So geht also Hochherrschaftl: gnädigster Befehl dahin, dass der Herr Pfarrer zu vigiliren (wachsam zu sein) und dahin bedacht

1 Dieser und der folgende Brief lagern im Archiv der Stadt Kirchberg/Jagst in Gaggstatt, Signatur A 116. Die Groß- und Kleinschreibung ist in diesem und den nachfolgenden Dokumenten sehr uneinheitlich. Zur besseren Lesbarkeit wurde die Großschreibung an die heutigen Gepflogenheiten angepasst. Der ganze Vorgang wurde - soweit ich sehe bisher einmalig - aufgearbeitet in: Franz Berger: Kirchgemeinde Großallmerspann 300 Jahre. Großallmerspann 1996, S. 41-44. Franz Berger war Lehrer in Großallmerspann; einer seiner Vorfahren war ein Bruder des hier genannten Michael Berger (Mitteilung von John Bachtler in Glasgow). Pfarrer Schäfer muss damals ganz neu in der Gemeinde Gaggstatt gewesen sein. Laut Pfarrtafel in der Kirche in Gaggstatt war er dort von 1747 bis 1762 Pfarrer. 
zu seyn habe, umb mit dem Tauf-Actu dem P(ater) zu Allmerspannt, wo möglich, bevor zu kommen. Sollte aber dieser etwa bey nächtlicher Weyle, wie allerdings $z u$ vermuthen, in das Bergerische Hauß einschleichen wollen, und das diesseitige Absehen nicht zu verrichten seyn, so wäre jedoch die Gelegenheit zu nehmen, um selben bey seiner Rückkehr auf öffentlicher Gaßen mit dem mündlichen Widerspruch ferner zu begegnen und aller weiterer rechtsbefugter Nothdurft vorzubehalten. Kirchberg, den 11ten Febr: 1747, Consistorium

Also: Der evangelische Gaggstatter Pfarrer soll aufpassen, dass er das Bergersche Kind taufen kann, bevor es der katholische Groß-Allmerspanner Pater tauft; wenn das nicht gelingt, soll er beim Groß-Allmerspanner Pfarrer protestieren und rechtliche Schritte der Kirchberger vorbehalten.

Um die Taufe durch den Gaggstatter Pfarrer durchzusetzen, ist die Hohenloher Seite sogar bereit, Waffengewalt einzusetzen. Im nächsten Brief an den Pfarrer in Gaggstatt heißt es: Im Fall aber dem Herrn Pfarrer (nämlich dem Gaggstatter Pfarrer) die Admission in das Bergerische Haus, zu bedeutem Ende, gleich wohl difficultiert werden wollte (der Zutritt zum Bergerschen Haus verwehrt werden sollte), so müßte bey Zeiten von der Sach Umstände Nachricht hierher gegeben werden, weilen die Hohe Hochfürstl. Absicht dahin geht, durch einiger abzuschikender Mannschaft Ihnen zu assistieren, damit Er seinen Zweck erreichen möge. Bey allem muß aber obgemelter Schultheiß nebst noch ein brav Mann von hiesigen Unterthanen, als Gezeugen, zugegen seyn. Und wird man sich also nach allem obigen zu richten haben. Kirchberg, den usw.

Was geht hier vor? Es soll - wenn notwendig mit Waffengewalt - verhindert werden, dass ein katholischer Pfarrer ein Kind katholischer Eltern tauft; und erreicht werden, dass es von einem evangelischen Pfarrer getauft wird. Geht es darum, eine Kinderseele der katholische Kirche vorzuenthalten und dem evangelischen Glauben zuzuführen? So erscheint es dem heutigen Leser dieser Briefe. So verhält es sich aber nicht.

\section{Mistlau - ein Ganerbiat?}

Dazu muss einiges zur damaligen politischen und kirchlichen Situation im Dorf Mistlau gesagt werden. Mistlau war seit dem Mittelalter bis zur Übernahme durch das Königreich Württemberg unter Napoleon von Untertanen dreier verschiedener Herrschaften bewohnt. Von den insgesamt 19 Höfen gehörten neun zu Comburg, neun zu Kirchberg und einer dem Haus Crailsheim bzw. dessen Nachfolgern, dem Haus Seckendorff ${ }^{2}$. Eine solche Konstellation war für die da-

2 Belegt z.B. im „Seelen-Register“ des Gaggstatter Pfarrers Rumpf aus dem Jahr 1802. Landeskirchliches Archiv Stuttgart, Pfarrarchiv Gaggstatt Nr 59 n. Demnach bestand Mistlau aus 19 Haushalten mit insgesamt 112 Einwohnern, wobei als Mitglieder eines Haushaltes Eheleute, Söhne, Töchter, Knechte, Mägde, Wittwer und Witwen gezählt wurden. 50 Jahre vorher dürften die Verhältnisse kaum anders gewesen sein. Von diesen 19 Haushalten zählten 9 zu den RitterStift Combur- 
malige Zeit nicht ungewöhnlich. Sie wird üblicherweise als Ganerbiat bezeichnet - als gemeinsame Herrschaft verschiedener Herren über ein Gemeinwesen ${ }^{3}$. Die Besonderheit in Mistlau liegt nun darin, dass sich die Partner nie über eine gemeinsame Verwaltung des Dorfes geeinigt, sondern jahrhundertelang über die Vorherrschaft über das Dorf gestritten hatten. Schon zur Zeit der Drei-Städte-Herrschaft (Dinkelsbühl, Rothenburg o.d.T. und Schwäbisch Hall hatten das Amt Kirchberg 1398 von Hohenlohe gekauft und 1562 wieder an Hohenlohe verkauft) gab es einen Prozess vor dem Reichskammergericht über die Frage, wem die Vogtei über Mistlau zustehe $e^{4}$. Das setzte sich bis zum Ende der Hohenloher und Comburger Herrschaft unter Napoleon fort mit unzähligen Briefwechseln zwischen den beiden Kontrahenten nach jeder Wirtshausschlägerei über die Frage, wer nun die Übeltäter aburteilen dürfe, bis hin zu einem Prozess vor dem Reichskammergericht in Wetzlar ${ }^{5}$. Geklärt wurde die Frage nie - ein Vergleichsvorschlag des Reichskammergericht im Jahr 1802 kam nicht mehr zum Tragen, da Württemberg 1802 die Comburger und 1807 die Hohenloher Herrschaft beendete.

Die beiden starken Partner in diesem Triumvirat - die Crailsheim-Seckendorffische Seite spielte praktisch keine Rolle - die Comburger und die Hohenloher, hatten denn auch je einen Schultheißen im Dorf Mistlau. Außerdem gab es zu der Zeit, von der hier berichtet wird, auch getrennte Wirtshäuser - eines für die Kirchberger Untertanen und eines für die Comburgischen Untertanen. Noch komplizierter wurden die Verhältnisse nun dadurch, dass Comburg katholisch, Hohenlohe und Crailsheim evangelisch waren. Mistlau selbst gehörte zur Pfarrei Gaggstatt; diese war 1556/57 mit dem Bekenntnis der Hohenloher Herrschaft zur evangelischen Religion evangelisch geworden. Damit war auch die Kirche in Mistlau evangelisch geworden samt allen Mistlauer Einwohnern.

gisch, jetzt herzogl. Wirtemb.Unterth. und Schutzverwandte(n); 9 zu den Fürstl. Hohenloh-Kirchbergische(n) Unterthanen und Schutzverwandte(n); 1 Haushalt zu den Freih. v. Sekendorfische Unterth.en und Schuzverwandte(n). Die 9 Höfe in Comburger Besitz bestätigt auch Berger (wie Anm. 1), S. 41.

$3 \mathrm{Im}$ 16. Jh. finden Ganerbiatstage statt, auf denen sich die drei Besitzer über strittige Fragen einigen. Dies endet mit dem Ganerbiatstag von 1611. Hohenlohe-Zentralarchiv Neuenstein (HZA) Ki 10 Nr. 13/Lit. B/31 f. Kirchberg verwendet danach den Begriff Ganerbiat nicht mehr, da es die Vorherrschaft in Mistlau beansprucht; Comburg dagegen nutzt ihn ausdrücklich - so auch im Revers, den Pfarrer Schäfer in Würzburg 1747 unterschreiben muss (s.u.).

4 Urteil von 1539 und 1566.

5 (Der erste Prozess endet mit einem Urteil vom 30. März 1688. Den zweiten Prozess strengt Hohenlohe im Jahr 1698 an. Er ist nie beendet worden.) Eine historische Aufarbeitung dieses Prozesses steht noch aus. Mandat des Reichskammergerichts vom 30. März 1688, Widerspruch Kirchbergs und weitere prozessuale Auseinandersetzungen bis 1700 . 


\section{Rekatholisierung durch Comburg}

Diese religiösen Verhältnisse waren nun bis 1681 stabil gewesen. Danach begann das Stift Comburg unter der Führung des Dekans von Ostein zunächst in Groß-Allmerspann und danach auch in Mistlau seine Güter mit katholischen Untertanen zu besetzen. Das war möglich durch das damalige System des Erblehentums oder Erbzinsgutes, das damals herrschte. Peter Steinle beschreibt dies so, „dass der Bauer zwar an seinem Hof nicht das volle Eigentum besaß, denn dieser (sic) lag in Form eines, Obereigentums ' bei der Herrschaft, dass er aber ein vererbbares dingliches Nutzungsrecht an dem Hof sein eigen nennen konnte." 6 Praktisch sah das so aus: Der Bauer konnte den Hof bewirtschaften, als sei er sein Eigentum. Wenn er starb, konnte er seiner Witwe oder seinen Nachkommen den Hof vererben. Dazu brauchte es allerdings die Zustimmung des Lehensherrn. Diesem mussten die Erben auch huldigen und einen „Handlohn“ - entsprechend unserer Grunderwerbssteuer - bezahlen. Im Hohenloher Landrecht war geregelt, dass dem Bauern und seiner Familie das Gut nur bei schwerwiegenden Verfehlungen entzogen werden konnte ${ }^{7}$. Ob es in Comburg ähnliche Regelungen gab, konnte ich nicht in Erfahrung bringen. Dieses Erblehensrecht hat nun seit 1681 das Stift Comburg benutzt, um zunächst in Groß-Allmerspann und dann auch in Mistlau auf seinen Gütern die evangelischen Besitzer zu verdrängen und durch katholische zu ersetzen.

Fürst Heinrich Friedrich Graf von Hohenlohe-Langenburg beklagt sich in einem Brief vom 30. November 1685 an den Bischof zu Würzburg ${ }^{8}$, dass der Augsburg. Confessions Verwandten (= evangelischen) Underthanen hinderlaßene armer Weib und Kinder, mit waß für Threnen undt Wehklagen ist leicht zu erraten, nicht anderst al $\beta$ coacti (gezwungen) zu emigriren, undt die lares paternos (den heimatlichen Herd), bloß darumben, weilen sie die Catholische Religion nicht annehmen wollen, schmertzhaft zu verlaßen angehalten werden.

Das Stift Comburg stellte also, wenn ein Haushaltsvorstand gestorben war, die Witwe vor die Wahl, entweder katholisch zu werden oder einen Katholiken zu heiraten oder das Gut zu verlassen und an einen von Comburg ausgewählten katholischen Nachfolger zu verkaufen. In Groß-Allmerspann besaß Comburg alle 14 Güter; kirchlich gehörte Groß-Allmerspann zur benachbarten evangelischen Pfarrei Lendsiedel. In Groß-Allmerspann hat Comburg innerhalb von 15 Jahren 13 der 14 Güter mit Katholiken besetzt. In der Folge wurde in Allmerspann vom Ritterstift Comburg auch eine katholische Kapelle gebaut und 1695 eine ständige Pfarrei eingerichtet und besetzt. Gegen all dies protestierte der Fürst von Hohenlohe-Langenburg als Kirchenherr von Lendsiedel in der Zeit

6 Peter Steinle, Die Vermögensverhältnisse der Landbevölkerung in Hohenlohe im 17. und 18. Jahrhundert, Schwäbisch Hall 1971, S. 43.

7 Ebd., S. 43.

8 StAL, B 375 L, Bü 647, Bericht Pfarrer Emer an Bischof von Würzburg vom 16. August 1747. 
von 1685 bis 1697 vehement; offenbar ohne Erfolg. Im Jahr 1685 wurde auch die Mühle in Mistlau mit einem katholischen Comburger Untertanen besetzt; ebenso eine zweite Hofstatt, in welcher zur Zeit unseres Berichts die Familie Berger wohnte.

Während die Hohenloher den Ort Großallmerspann offenbar ab 1700 verloren gegeben hatten, entspann sich um die Herrschaft in Mistlau nun ein zäher Kleinkrieg, der bis in die napoleonische Zeit andauerte. In der Zeit von 1698 bis 1747 köchelte der Religionskrieg zwischen Comburg und Hohenlohe auf kleiner Flamme. Inzwischen war Kirchberg innerhalb des Hohenlohischen Familienverbandes $1701 \mathrm{zu}$ einer selbständigen Grafschaft geworden; vielleicht war die neue Verwaltung zu sehr mit diesen und anderen Dingen beschäftigt, um sich mit Comburg um die pfarramtlichen Rechte in Mistlau zu streiten. Der katholische Pfarrer Emer beruft sich jedenfalls in den folgenden Auseinandersetzungen darauf, er habe 14 Jahre lang ungehindert in Mistlau actus parochiales - pfarramtliche Akte - ausführen können?

\section{An einer Taufe soll ein Exempel statuiert werden}

Im Jahr 1747 aber flammte der Streit plötzlich auf. Offenbar wollte Hohenlohe-Kirchberg die Taufe eines Bergerschen Kindes zu einem Präzedenzfall machen. Wie das vor sich ging, ist am bestem dem Bericht des katholischen Pfarrers von Allmerspann mit Namen Emer vom 16. August 1747 an die Kanzlei des Fürstbischofs in Würzburg zu entnehmen.

[...] den 4. April thate mir Michael Berger die Anzeig wie dass sein Weib des Kinds niedergekommen, und also ich denselben Tag noch die $h$. Tauff mittheilen mögte:

ginge darnach nach vollender Vesper fort, bey Klein-Almerspan aber kame mir schon entgegen geloffen des besagten Bergers Knecht, der mir andeutete, der lutherische Pfarrer zu Gaggstatt wolle das Kind taufen; zu dem End stehe derselbe samt dem Hohenlohischen Schultheißen und etlichen Unterthanen bey dem Steg zu Mistlau, dass selbe mir im Nahmen Ihres Principals in faciem resistiren (ins Angesicht widerstehen) und den acceß (Zugang) hindern wollten; diesem ohngeacht sezte ich meinen Weg fort, schickte aber den Knecht voraus, dem Comburg: Schultheißen zu bedeuten, dass er mich manuteniren (zur Hand gehen) sollte; Treffe also bey Mistlau an den Pfarrern zu Gaggstatt samt einigen Hohenlohischen Unterthanen die gegen mich solemnissime (feierlichst) protestirt:

Den Protest wies der Pater zurück: ginge also ohn weiteren Widerstand dem Comburg. Hauß zu: kaum ware ich in dem Hauß, dann dahergeritten der Kir-

9 Ebd. 


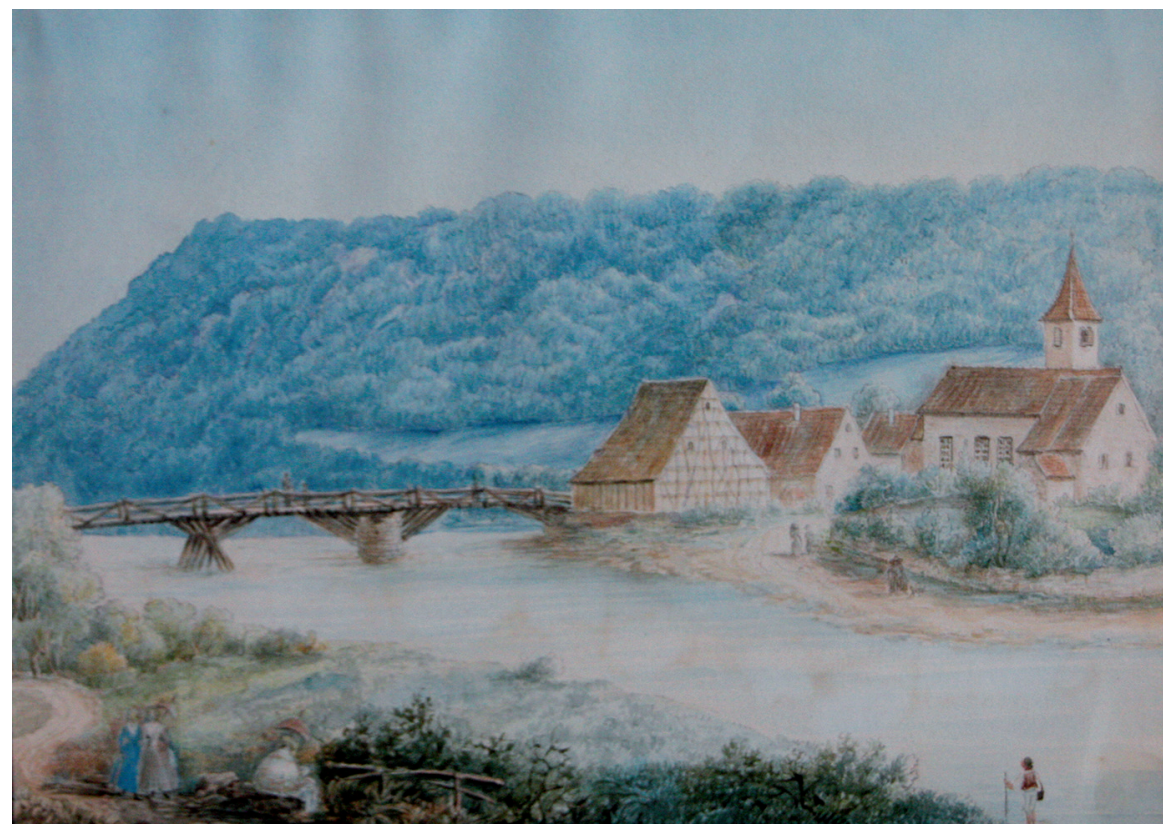

Abb. 1: Mistlau mit Kirche und Steg um 1840 (Aquarell von Marie von Hohenlohe-Kirchberg.Original im Sandelschen Museum in Kirchberg.

Foto: Ulrich Fröhner)

chbergische Cantzley-Secretarius mit 11 Mousquetiers und einem Corporal sprange vom Pferd und wollte eylends in das Haus des Bergers eindringen, wurde jedoch von dem Comburg. Schultheißen zurückgehalten, so lang bis der Tauff-actus vorbey ware.

Danach betrat der Kirchberger Kanzlei-Sekretär das Haus, protestierte beim Pater gegen diesen Tauf-Akt und verlangte von diesem einen Revers, eine Bescheinigung, dass er unrechtmäßig gehandelt habe. Das verweigerte der Pater und sagte, er würde das, wenn notwendig, auch dem Grafen selbst ins Gesicht sagen. Prima, sagte der Kanzlei-Sekretär - genau darum geht es - er möge doch mit nach Kirchberg kommen.

Da ich aber sagte, ich wolte nur zuvor den gnädigen Specialbefehl von meinem Hohen Principal S. Hochwürden und Gnaden H. Dechanten zu Comburg erwarten, da befahle er denen Soldaten - denen die Zähn nach meiner Haut gewässert-das Hauß rings um wohl zu verwahren, bis auf weithere Verfügung, worauf ich geantwortet, es gibt vielleicht in dem refier Wirtzburgische Husaren, die mich werden redimiren (herausholen); Nachts um 9 uhr bekam der commandierte Corporal ein Schreiben hujus tenoris (dieses Inhalts): Er Corporal sollte dem H:en Pater ein schönes Compliment machen und bedeuten, er mögte 
doch nur mit dem Corporal allein auf Kirchberg gehen, und dort wegen seines Unternehmens mit H.en Graffen sprechen: welches als ich gelesen, stellte solches in fail (in Frage) und sagte, dass ist ein Narrheit von denen Beamten, davon weiß der H: Graff nichts: überschickte dann diese Schrifft nebst meiner relation (Bericht) selbige Nacht noch auf Comburg. Des andern Tags nun 11 uhr ruckte eine stärkere Mannschaft an von Bürgern und Soldaten, Amtsknecht und Büttel cum gladys et fustibus (mit Schwertern und Fäusten) unter Anführung des Rathes und Amtmanns von Kirchberg, welchen dann mit denen Soldaten - da ich weder freywillig mitgehen, noch auch einen Revers ausstellen wollte - mit großen Tumult und Geschrey gewaltsamer weiß in das Hauß eingefallen und mich von der Seithen der kranken Kindsbetterin hinweggenommen. Der Pater wurde nach Kirchberg geführt unter großem Zulauff des gemeinen Pöbels so wohl in Mistlau als auch und besonders in Kirchberg: allwo ich ohne bey dem H:en Graffen Audienz zu haben, [...] in das so genannte stättlich Wirthshau $\beta$ von dem hierzu bestelten Cantzley Secretario einlogiert worden bin, worauf vermelter Secretarius ein gewiße Method eines Reverses produciert, selbe mir zu lesen gegeben, und dann selbe abzuschreiben blandytis ac minis (mit Drohungen und Schmeicheleien) unter ungestümer Beystimmung des Raths und Amtmanns mich zu vermögen gesucht.

Der Pater lehnte es ab, den Revers zu unterschreiben und verwies auf die Rechte des Stiftes Comburg und des Bischofs von Würzburg.

Nach diesem wurde mir in obbesagten Stättlis-wirthshaus das sogenannte jemahlige Gerichtsstüblein eingeräumet, allwo (ich) greülich durchlögerte Fenster und abscheülich unsauberes Beth angetroffen und darum auch die 2te nacht keinen Schlaff gehabt den dan (welcher ich) nebst dem rauhen Nachtlufft, Alteration (Aufregung) und Schröcken wegen der kranken Kinsbetherin mein noch würklich andauernde maladie am rechten arm und hand zuschreibe, an welcher (ich) 2 Finger dato noch nicht recht brauchen kann, wie ich dan auch dessentwegen noch immer mit Medicin anhalten muß.

Am folgenden (Freitag) Morgen ging Pfarrer Emer zum Kanzleidirektor Ulmer und verlangte ein besseres Quartier. Es wurde ihm erlaubt, in der Wohnung des Hofverwalters zu schlafen und im Wirtshaus zu essen. Am Samstagmorgen machte er einen kollegialen Besuch beim evangelischen Hofprediger. Von dort wurde er auf die Kanzlei zitiert; dort versuchten Kanzlei-Direktor, Amtmann und Kanzlei-Sekretär mit vereinten Kräften, ihn doch zur Unterschrift unter einen Revers zu bewegen. Als er standhaft blieb, teilten sie ihm mit, dass vom Grafen am Abend vorher ein Schreiben eingegangen sei mit der Erlaubnis, ihn zu entlassen; doch solle er die Kosten für seine Verpflegung im Wirtshaus ersetzen - auch das verweigerte Pfarrer Emer standhaft. Darauf wurde der Pater noch einmal verhört und ihm danach seine Entlassung mitgeteilt. Dies liest sich so: Es wird hiemit der H. Pater in Regard (Berücksichtigung) des Ritterstiffts Comburg und um die Nachbarschafft zu pflegen in Gnaden entlassen, demselben aber mit allem Ernst verbotten inskünfftig die unstrittige höchste Bischöfl. jura 
S:er Excellenz H:en grafens etc. nimmermehr zu kränken und keinen actum parochialem mehr in Mistlau ohne requisition (Erlaubnis) zu unternehmen ${ }^{10}$. Damit war das Abenteuer für Pater Emer zu Ende.

\section{Keine Glaubensfrage}

Warum geht es hier? Es geht also nicht darum, ob das Kind katholisch oder evangelisch getauft wird, sondern darum, wer die Taufe vornehmen darf - ob der katholische Pfarrer als Vertreter der Herrschaft des Stifts Comburg oder der evangelische Pfarrer von Gaggstatt als Vertreter des Grafen von Hohenlohe. Die hohenlohische Seite wäre damit zufrieden gewesen, wenn der Pfarrer von Groß-Allmerspann die grundsätzliche (pfarramtliche) Herrschaft des Grafen von Hohenlohe eingestanden hätte - und hätte dann nichts gegen die katholische Taufe einzuwenden gehabt. Auf eine solche Lösung hat man sich tatsächlich nach sieben Jahren Streit auch geeinigt (s.u.). Es ging hier also nicht um eine Glaubens-, sondern um eine Machtfrage. Darauf wird weiter unten noch näher einzugehen sein. Aber sehen wir zuerst, wie die Geschichte weiterging.

Zunächst löste der Vorfall einen heftigen Schriftwechsel zwischen dem Stift Comburg und der bischöflichen Kanzlei in Würzburg einerseits und der Hohenlohe-Kirchbergischen Kanzlei andrerseits aus. Sofort nach dem Vorfall protestierte Comburg natürlich schriftlich in Kirchberg. Gleichzeitig informierte es die bischöfliche Kanzlei in Würzburg. Von dort kam am 11. April 1747 ein Antwortschreiben an die Kanzlei in Comburg. Würzburg forderte nähere Informationen; gleichzeitig mahnte es zur Vorsicht: das Jus ecclesiasticum (das bischöfliche Recht, das Würzburg zustehe) müsse nicht zwangsläufig auch das Jus parochiale (das Recht über das Pfarramt) umfassen. Es wurde auf Fälle verwiesen, in denen Würzburg seinerseits das Jus parochiale in evangelischen Gebieten beanspruche. Von der juristischen Fakultät der Universität Würzburg wurde ein Gutachten angefordert, das am 17. November 1748 abgeliefert wurde.

Dem Pfarrer Emer wurde seine Standhaftigkeit in Würzburg übrigens nicht gedankt. In einem Schreiben Würzburgs an Comburg vom 8. April 1747 (also vier Tage nach der Festnahme des Pfarrers) schreibt ein F.W. Habermann, erstens sei am Samstag keine Sitzung der Regierung, deshalb könne man sich erst kommenden Montag mit der Sache befassen; und zweitens hätte man sich einiges ersparen können, wenn Pfarrer Emer den Revers unterschrieben hätte - das wäre dann seine Privatsache gewesen und deshalb rechtlich nicht bindend, und widerrufen hätte man den Revers immer noch können ${ }^{11}$.

10 Ebd.

11 Alle diese Dokumente StAL, B 375 L, Bü 647. 


\section{Das Imperium schlägt zurück}

An der „Front“ scheint es inzwischen ruhig gewesen zu sein. Allerdings nur bis zum 9. April 1748. Da nämlich wurde im Hause Berger - fast genau ein Jahr nach der ersten Geburt - wieder ein Kind geboren. Und diesmal war die Kirchberger Seite schneller. Nach Darstellung der Comburger Seite hatte der Gaggstatter Pfarrer Schäfer mit Beyhülf zehen Gräfl. Hohenlohe-Kirchbergischer bewöhrter (bewaffneter) Mannschaft das neugeborene Kind aus dem Bergerischen Haus heraus durch einige hohenlohische Frauen in dasige lutherische Kirche tragen [...] lassen und daselbst den Tauf-Actum des Kindes[...] vorgenommen."

Comburg berichtete dies am 13. April 1748 an die Kanzlei des Fürstbischofs in Würzburg (zu dessen Herrschaft Comburg gehörte) und schlug vor, so wie Kirchberg den Pfarrer Emer verhaftet hatte, nun den Pfarrer von Gaggstatt in Arrest zu nehmen. Würzburg ließ sich damit noch zwei Monate Zeit, schlug dann aber umso härter zu. Was geschah, liest sich im danach erfolgten Protestschreiben der Kirchberger Kanzlei so ${ }^{12}$ :

\section{Juli Anmarsch}

Eine vollständige Kompanie Würzburger Dragoner - das sind ungefähr 70 Mann - zog in der Nacht vom Samstag auf den 30. Sonntag nach Trinitatis 1748 - das war der 12./13. Juli - von Groß-Allmerspann nach Gaggstatt, und zwar über Dörrmenz, Eichenau, das Breitloh, die Weckelweiler Steige hinunter, unten am Schloss vorbei sogar unterhalb der hiesig Hochgräflichen Residenz selbsten, was für ein Affront! - und dann nach Gaggstatt hinauf, wo selbst in der mitternacht umb 1 Uhr nicht allein der disseitig ohnstrittig Pfarr-und Kirchhof zu Gaggstatt sogleich besezet, sondern auch die Pfarr Wohnung selbst, nach Niderreisung eines Stückhs des hinteren gartenzaunes, unmittelbahr völlig umbringt, und so fort dem durch sothane feindliche Anstalt, aus dem ersten Schlaaf erweckhten des ganzen Vorgangs und Absehens im mindesten nicht vermuthend gewesenen, und sich auf die Predigt des bemelten Sonntags und den haltenden Gottesdienst in seiner Pfarrkirche preparirt gehabten Pfarrer Schäferen von dem commandierenden Herrn Haubtmann von Nassau unter bedroheter Gewalth zugerufen worden, das Haus zu öffnen. Da nun Er, H. Pfarrer, in der äussersten Consternation, worinne Er sich durch ohnvermutheten Überfall gesezet gesehen, anfänglich wider die Gewalth protestirt, und zu Öffnung des Pfarr-Hauses sich so bald nicht entschliessen können, in deme er sich gar nicht dazu stellen gewusst, woher dergleichen Vergewaltigung gegen Ihn, zumahlen in offenen Friedens Zeiten, kommen solle, sind sogleich auf Ordre des Commandirenden Officiers einige seiner Leuthe, mittelst eines vor dem Hof gele- 
genen grossen Balkens wider die Thür des Pfarrhauses dergestalt gewaltsamb angerennet, dass dadurch der Rigel gesprenget worden, und sich solchem nach der Pfarrer ergeben müssen."

Dem Pfarrer wurde vom mit angerückten Comburger Kommissar Kleiner mitgeteilt, er sei hiermit verhaftet. Nun weiter im Bericht aus Kirchberg: Da er (der Pfarrer) inzwischen nach angehörter Arrest Ankündigung kaum so viel Zeit übrig gehabt, sich nur in die benötigte Ankleidung zu sezen, ja sogar auch wehrend dieser Beschäftigung Ihm eine Wache mit bloßem Pallasch (Säbel) vor die Studir Stube gestellet, und Er sodan des kläglichsten Lamentirens seiner Frau ungeachtet sogar anfänglich ohnwissend und zweifelhaft mit wem Er es desshalben zu thun habe, nicht nur alß ein Malefiziant (Übeltäter), sondern auch weg des unbekanthen Commando und vieler Mannschaft, alß ein von frembdem Kriegs Volckh in Mitternacht überfallener und vergewalthättigter Mann hinab in den Hof geführt, auf ein davor gehaltenes Pferd zu sitzen angewiesen, und genöthiget worden, sodan in der eitlen Nacht, und zwar in beständig Regen Wetter den Zug durch Mistlau nach grossen Allmerspann fortzusetzen, und er daselbst den Mittag über in strackhem Arrest behalten worden. Bey dem Abmarch von da ist er, H. Pfarrer, zwar wider zu Pferd gesezet, dises aber zu nicht geringer seiner Prostitution einem Bauern zu führen überlassen, und solch elendem Aufzug er in der Mitte der Comapgnie unter gerührter Trommel, durch das zusamb gelaufene und frohlockende Catholische Volckh, zum Schauspiel ab und nach Comberg geführet worden.

\section{Weitere Aktionen}

Es ging also von Groß-Allmerspann noch am selben Tag weiter nach Comburg bei Schwäbisch Hall. Von Gaggstatt über Mistlau nach Groß-Allmerspann sind es ungefähr $10 \mathrm{~km}$, weiter nach Comburg noch einmal $20 \mathrm{~km}$. Dort wurde der Pfarrer im Haus des dortigen Posthalters arrestiert unter Bewachung von einem Unteroffizier und sechs Dragonern. Er erhielt auch etwas zu essen und es wurde ihm erlaubt, seiner Familie Nachricht zu geben. Dienstag, 16. Juli 1748, erfolgte dann um 22:00 Uhr der Weitermarsch.

Vergeblich hat er gefragt, wohin mann Ihn denn weiter bringen wollte? da Er in den Stiftshof geführt, allda wieder von einem Commando, in einem Unterofficier und 6 Gemeinen bestehend, angenohmen, zu Pferd gesezet, und auf obbeschriebene unanständige arth durch einen Botten, dem der zügel des Pferdts zugestellet wurde, mit gröster Lebensgefahr in der stockhfinstern Nacht durch lauter Umb- und Neben-wege bis nacher Mulfingen ungefehr fruhe gegen 8 uhr unter abermahligem zulauf des gereizten höhnischen Pöbels zu nicht geringer Prostitution überbracht.

Von Comburg bis Mulfingen sind es etwa $30 \mathrm{~km}$. Der Zug muss Um- und Nebenwege nehmen, um so weit möglich hohenlohisches Gebiet zu vermeiden. In Mulfingen wurde der Pfarrer zum dortigen Centschultheißen (Gerichtsschulthei- 


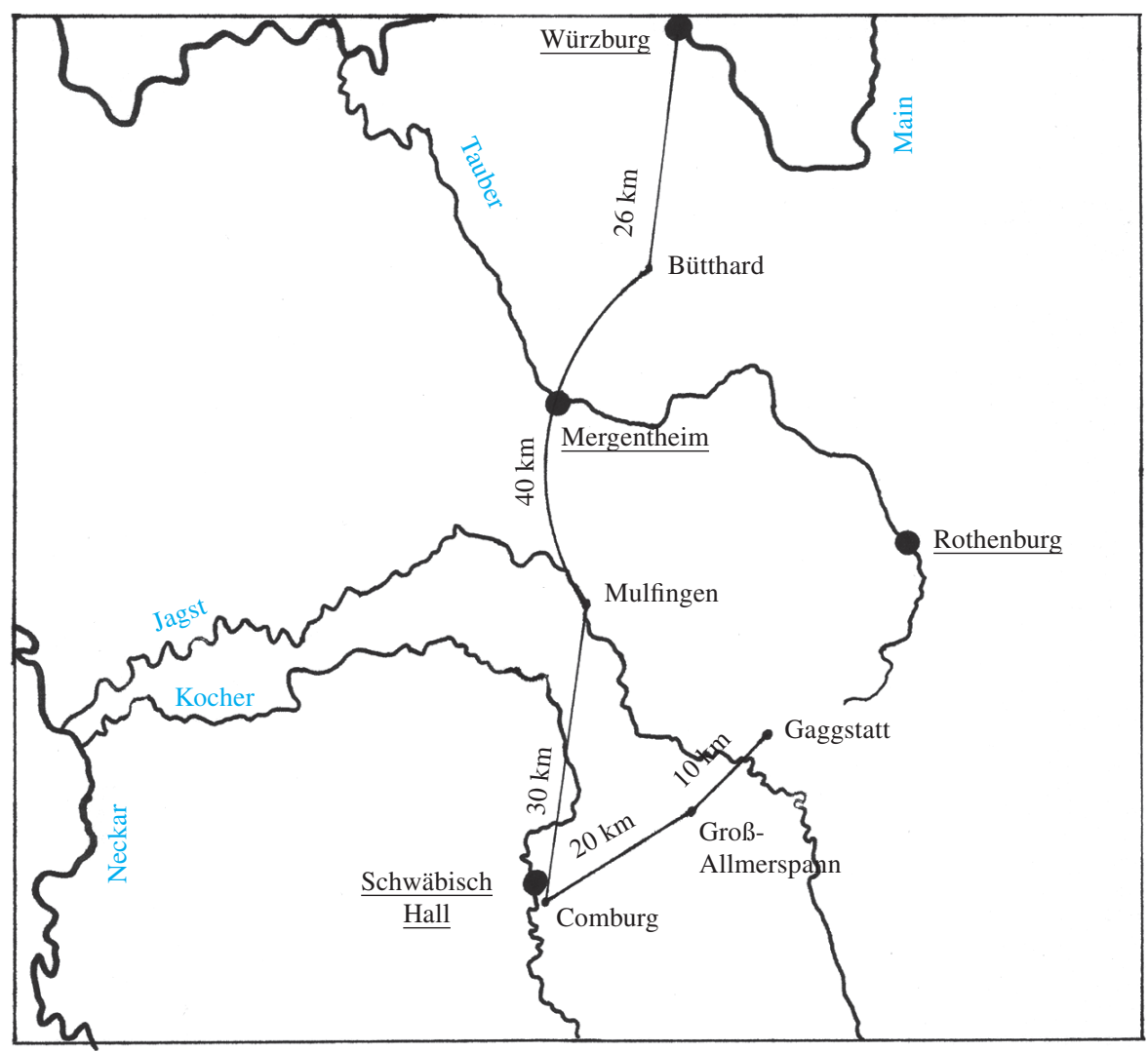

Abb. 2: Die Entführung von Pfarrer Schäfer 1748 von Gaggstatt nach Würzburg (Entwurf: Ulrich Fröhner, Ausführung: Gerhard Fritz)

ßen) geführt, der ihm ein Quartier zuwies. Um die Mittagszeit fragte er nach Essen; er erhielt zur Antwort, wenn er Geld habe, könne er etwas bekommen. Er bat darum, einen Boten nach Hause schicken zu dürfen, um Geld und Wäsche bringen zu lassen. Das wurde ihm abgeschlagen.

Am selben Abend, Mittwoch, 22:00 Uhr, brach der Zug wieder auf, Pfarrer Schäfer wieder zu Pferd. Es ging über Mergenthal (Bad Mergentheim) nach Büttert (Bütthard). Das ist eine Entfernung von ca. $40 \mathrm{~km}$. Dort langte der Zug am Morgen des 18. Juli an.

Bey dieser abermahligen Station nun hatte er (Pfarrer Schäfer) sich eben so wenig Comodität (Bequemlichkeit) als vorher zu versprechen, dabey aber sich von Kräften so sehr verlassen gefunden, dass er geglaubt er würde endlich unterligen müssen. Doch hat ihn Gott soweit in seinem Gemüth bey seinem unschuldigen schwehren Leyde gestärckhet, um noch härtere Fatiguen (Erschöp- 
fungen) und Drangsalen ausstehen zu können. Diese Erschöpfungen und Drangsalen bestanden zunächst aus einem weiteren Marsch am gleichen Abend 23 Uhr, nämlich von Bütthardt nach Würzburg, das sind rund $25 \mathrm{~km}$. Und diesmal musste er zu Fuß gehen. Dies wurde von Kirchberg natürlich als zusätzliche Schikane dargestellt. Denkbar ist aber auch, dass nach einem Ritt von insgesamt $100 \mathrm{~km}$ innerhalb von 6 Tagen bzw. Nächten ein weiterer Ritt dem Sitzleder von Pfarrer Schäfer überhaupt nicht mehr zugemutet werden konnte.

Ankunft in Würzburg Samstag 20. Juli 7 Uhr in der Fruhe: Über alle ausgestandene harte Strapazen hat er bey fast gänzlich verlohrenen Cräften noch die Schmach erdulden müssen, daselbst als ein Missetäter unter grossem Zulauf des Pöbels eingeführt, und auf die Haubtwache, ja so gar an eine solche Gelegheit gesezet zu werden, da er an denen an der Wandt bevestigten Ketten und Globen zu seinem kaum überwindlichen Erstaunen und Horrore mehr als zu sehr wahrnehmen auch sonst erfahren müssen, das solche seine Behaltnus, wo arme zum Todt verurtheilte Missetäter, usque ad supplicium (bis zur Hinrichtung) bewahrt zu werden pflegten. Die ihm daselbsten angewiesene Lagerstatt ist in einer leeren Pritsche bestanden. Zur Wache aber ein Soldath mit aufgepflanztem Bajonet Ihm vorgestellet worden. Des unerträglichen Gestanckhs den er in disem Gefängnus auszustehen gehabt, nicht erst zu gedenckhen.

Er wurde noch am Morgen auf die Kanzlei geführt und verhört: Allwo er leediglich über den lezthin an dem Bergerischen Kindt zu Mistlau vorgenohmenenTauf Actum constituiret, und Ihme darbey bedrohlich zugemuthet worden, dass er sich dieserwegen noch selbig Tags einen revers zu unterschreiben bequemen, oder widrigenfalls sich selbst beymessen müste, wen der Arrest die Ferien über gegen Ihn verhänget bleibe, in deme wehrender solcher in Zeit von 3 Wochen nichts vorgenohmen würde.

Um 17 Uhr wurde er zu einem weiteren Verhör geführt. Jetzt wurde die Feriendrohung noch ausgedehnt auf vier Wochen. In Erwägung nun das seine Freyheit anderergestalt nicht wieder zu erhalten, er aber nach so vielen bereits erlittenen Drangsalen in obbeschriebenen Carceris squalore ohnfehlbar würde des Todtes gewesen, darunter der Sache aber ebenso wenig gerathen, als durch die abgenöthigte Unterschrift solcher einig praejudiz zu gefüget worden seyn, hat er sich endlich nolenter volenter darzu bequemet.

Pfarrer Schäfer hatte also den Revers unterschrieben und war darauf am Sonntagmorgen, 21. Juli aus der Haft entlassen worden. Man teilte ihm mit, er könne sich nun um seine Heimreise kümmern. Gegen den Vorwurf der schlechten Behandlung des Pfarrers Schäfer verwahrte sich Comburg später gegenüber Würzburg mit folgenden Worten: Was nun anbetrifft die wahre Beschaffenheit der Arrestirung des mehr erwehnten Pfarrers Schäfers zu Gaggstatt, wird dieselbe zum Theil ohnschwehr ab dem sub Lit. B hir beygehenden Bericht unseres Commisseres Kleiner zu entnehmen seyn, zum Theil aber sollen (wir) noch hier erwehnen, [...] was honetere Verpflegung so wohl unterwegs nacher Comburg, als dahier selbsten dieser Minister empfangen habe, als der Allmerspanner 
Pfarrer Emmer in seinem vormahligen Arrest zu Kirchberg, sintemahlen mann ihme unterwegs vom Tisch deren HH. Officiere hat angedeyhen, auch zu Comburg die Kost deren Vicarier hat abreichen lassen, und waar in summa das Tractament nicht so beschaffen, wie sonsten ein Arrestant gehalten zu werden pfleget, in deme ihme erlaubt war, sich mit honeten Leüthen in dem Zimmer zu unterhalten, bey seiner Hinwegführung aus Comburg hat derselbe wiederumb ein Pferdt bis nach Mulfingen empfangen, ... was sich im übrigen mit dem oft ermehlten (erwähnten) Ministro zugetragen, und mit selbem auch in Wirtzburg unternohmen worden, wird unseren Hochgeehrten Herren von selbsten wohlbekannt seyn.

Die Verhaftung des Pfarrers von Gaggstatt erregte großes Aufsehen. Natürlich protestierte Kirchberg sofort; dem Protest schlossen sich auch Schwäbisch Hall und Brandenburg-Ansbach an, deren Gebiet der Zug der Dragoner berührt hatte. Kirchberg verlangte in seinem Protestschreiben eine Entschädigung an Pfarrer Schäfer für den erlittenen Unbill und die Herausgabe des Revers, den Pfarrer Schäfer unterschrieben hatte. Würzburg ließ sich natürlich von Hauptmann von Nassau und Kommissar Kleiner schriftlich berichten und zunächst von Comburg eine Einschätzung zu den Vorwürfen der Kirchberger geben; daraus stammt die oben zitierte Verteidigung gegen den Vorwurf der schlechten Behandlung des Gefangenen. Würzburg bemühte weiter die Juristische Fakultät in Würzburg um ein Gutachten; auf die Forderungen Kirchbergs ging es natürlich nicht ein.

\section{Eine weitere Taufe}

Im August des folgenden Jahres 1749 stand dann die nächste Geburt im Hause Berger an. Comburg sah sich diesmal besser vor und blieb bei seinem martialischen Auftreten: diesmal wurden 50 würzburgische Soldaten aufgeboten, die alle Comburgischen Häuser in Mistlau besetzten. Kirchberg seinerseits hatte einen Notar mit zwei Kirchberger Bürgern beauftragt, das Ganze zu beobachten und zu protokollieren. Von Comburg wurde ein Kapuzinerpater geschickt, welcher das Kind taufen sollte. Der Notar Gebler von Kirchberg versuchte, diesen Pater am Steg in Kirchberg abzufangen ${ }^{13}$.

Nach Verlauf einer guten Stunde, nachmittags nach 2 Uhr, erhielten wir aber durch die auf Kundtschaft aus(ge)schickte Personen die Nachricht, dass ein Capuziner von Comburg, unter Begleitung Würzburgischer Mannschaft, einen ganz unbekannten Weg, von Lobenhausen her, oben gegen das Dorf zu, welchen man sonsten fast niemahls zu gehen pflege, hergegangen komme; wie denn einige Personen würcklich wahrgenommen hatten, dass gedachter Capuziner, mit der ihm zur Escorte dienenden Würzburgerischen Mannschaft, in Ermangelung 
eines ordentlichen Stegs, oben bey dem Mistlauer MühlWehr über den vorbey fliessenden Jagst-Fluß gewaden (gewatet?) war.

Der Notar versuchte, beim Kapuzinerpater zu protestieren, aber vergeblich - der Pater verschwand im Bergerschen Haus und taufte dort das Kind, während die Würzburger Eskorte das Haus umzingelte und ihm den Kirchberger Notar vom Leib hielt. Der Notar versuchte dann, beim kommandierenden Würzburger Offizier seinen Protest anzubringen; der aber wollte nichts hören. Und als ich deßen ohngeachtet, schreibt Notar Gebler in seinem Protokoll, den Anfang mit meiner Protestation machte, wurde von dem Herrn Hauptmann dem Tambour Ordre gegeben, er solle das Spiel rühren, jedoch nicht allzu stark, damit die Kindbetterin in dem Hause nicht davor erschrecken mögte. Dieser fing auch die Trommel an zu rühren, wie wohl solche von ihm mit so wenigem Laut geschlagen wurde, dass der Herr Hauptmann, die andern beyden Officiers, meine zwey Zeugen, und die umstehenden Personen, alle Worte meiner Protestation vernehmen können. Ich ließ mich darnach nichts abhalten, sondern legte solche Protestation vor denen Würzburgischen Officiers, insonderheit gegen den commandierenden Herrn Hauptmann, in Gegenwartt der mir zur Seite stehenden zweyen Zeugen, und der sämtlichen bewehrten bey 50 Mann starken Würzburgischen Miliz, so un $\beta$ umringet hatten, auch in Beysein von vielen anderen Personen, so als Zuschauer herbey kommen, ab, und thate auf offentlichem Plaz, unter freyem Himmel, in dem Dorfe Mistlau, vor des Comburgischen Hintersaßen Michael Bergers Haus, gegen den Würzburgischen Hauptmann folgende Proposition:[...]

Es folgt ein ausführliches Zitat des Protestes des Notars. Der Pater habe gegen die Territorial- und Episcopal-Rechte von Hohenlohe-Kirchberg verstoßen. Danach zogen beide Seiten vermutlich zufrieden ab - die eine, weil sie das Kind getauft hatte, der Kirchberger Notar, weil er seinen Protest losgeworden war. Über all das fertigte der Notar ein ausführliches Protokoll an ${ }^{14}$. Es beginnt mit der Standardformel solche feierlicher Urkunden:

Im Nahmen Gottes! Kund und zu wißen seye hiermit jedermämmiglich, durch dieses offene Instrument, dass im Jahr nach Christi, unseres Heilands, Geburt, eintausend sieben hundert neun und vierzig, ... Und dann wird berichtet, was hier Empörendes geschehen ist.

\section{Die dritte Taufe - und einige Todesfälle}

Und nun folgte ein Jahr Pause. Dann im Frühjahr 1751 kündigte sich bei Bergers eine neue Geburt an. Comburg fragte deshalb bei Würzburg an, wie man dieses Mal verfahren solle. Würzburg, dessen Vorgehen in den Vorjahren dem Fürstbischof und seiner Kanzlei offenbar doch eine sehr schlechte Presse eingebracht 
hatte, mahnte dieses Mal zur Deeskalation. Empfehlenswert sei, den Streit nicht zu verschärfen, sondern die Niederkunft auf comburgisches Gebiet zu verlegen und das Kind dort taufen zu lassen. So scheint es auch geschehen zu sein - jedenfalls hören wir von diesem Tauf-Actus oder einem Streit darum nichts mehr ${ }^{15}$. Dafür beschäftigen nun einige Todesfälle im Haus der Bergers die beiden Streitpartner. Am 23. April 1751 starb eines der Bergerschen Kinder (aliquot annorum = einige Jahre alt). Comburg schickte den Notar Kleiner mit zwei Zeugen, um die Leiche des Kindes nach Allmerspann zu überführen und dort begraben zu lassen. Die Kirchbergische Seite beschlagnahmte die Leiche aber sofort und gab sie erst frei, nachdem Notar Kleiner einen Revers (eine Bescheinigung) unterschrieben hat, der bestätigte, dass Kirchberg trotz Freigabe der Leiche an seinem Rechtsanspruch auf die Parochialrechte in Mistlau festhalte. Dieser Vorgang scheint der Comburgischen Seite peinlich gewesen zu sein - der Bericht des Notars Kleiner erhält am 20. April 1752 (ein Jahr später) den Vermerk: geheim. Am 3. Juni 1751 starb Catharina Margaretha, ein weiteres Kind von Berger, drei Jahre alt. Es war das Kind, das vom Gaggstatter Pfarrer Schäfer getauft worden war. Laut Aufstellung des Pfarrers von Groß-Allmerspann wurde es in Groß-Allmerspann begraben. Über irgendwelche Komplikationen dabei ist nichts bekannt.

Am 13. Dezember 1751 schließlich starb die Schwiegermutter von Michael Berger namens Weidner. Comburg schickte wieder Notar Kleiner, um zusammen mit dem Pfarrer von Großallmerspann die Leiche dorthin zu überführen. Der Bericht des Notars ist wieder sehr anschaulich, soll hier aber nur in Zusammenfassung wiedergegeben werden. Notar Kleiner erschien also am Donnerstag, nachmittags um 4 Uhr, mit zwei Zeugen in Mistlau. Er fand das Bergersche Haus von Kirchberger Soldaten umstellt, konnte aber keinen Kirchberger Beamten ausfindig machen. Am Freitag um 14 Uhr erfuhr er schließlich, dass der Kanzlei-Sekretär Conradi von Kirchberg sich im Haus des Kirchbergischen Schultheißen aufhalte. Er ging mit seinen Zeugen dorthin, protestierte gegen die Belagerung des Bergerischen Hauses und forderte die Herausgabe des Leichnams der Bergerischen Schwiegermutter. Conradi forderte umgekehrt wieder einmal einen Revers von Kleiner. Kleiner erklärte, er sei befugt, einen Revers auszustellen - aber nur mit dem Vorbehalt einer höchstrichterlichen Überprüfung. Dagegen hatte nun Conradi Vorbehalte - er sagte, er müsse diese Formulierung erst genehmigen lassen und sagte eine Antwort bis Samstag früh um 9 Uhr zu. Der Leichnam bleibe so lange noch im Bergerschen Haus. Am Samstag um 10:30 schickte dann Conradi zu Kleiner: es gebe noch etwas zu besprechen wegen des Revers. Es seien noch zwei Kleinigkeiten zu regeln: zum einen müssten dem Pfarrer zu Gaggstatt die Gebühren für eine - in diesem Fall entgangene - Beerdigung bezahlt werden; und zum andern müsse im Revers noch stehen, dass die Beerdigung auf keinen Fall den Rechten des Fürsten von Hohenlohe-Kirchberg 
Abtrag tue. Das lehnte nun wieder Kleiner ab - dazu habe er keinen Auftrag. Und nun im Original: unter dieser Zeit hat aber der Comburg. Unterthan Michael Berger seine verstorbene Schwieger Mutter zum Haus heraustragen auff einen Wagen laden, und mit forth nach Allmerspann fahren wollen, ist auch bis zum Endte des Dorffs, wo die Kirchen stehet, mit forth gekommen, und sind die Leüth ordentlich wie sonsten hinter dem Wagen noch mit zur Leicht $($ Leich = Beerdigung) gegangen, auff einmal aber sind daselbsten durch Kirchberg bewaffnete Burger dem am Wagen gestandenen Wirthe ins Geschirr gefallen, haben den Wagen mit Gewalt angehalten, und haben die Verstorbene herunter und in die dasige Kirchen gethan.

Natürlich protestierte Notar Kleiner wieder heftig, aber ohne Erfolg; die Bergersche Schwiegermutter wurde am 16. Dezember 1752 vom protestantischen Pfarrer von Gaggstatt auf dem Friedhof in Mistlau beigesetzt.

Inzwischen schwelte der Streit zwischen Kirchberg und Comburg auf Kanzlei-Ebene weiter. Zur Untermauerung seiner Ansprüche ließ Comburg den Pfarrer von Allmerspann eine Aufstellung machen über Trauungen und Begräbnisse von Mistlauer Einwohnern in der Gemeinde von Allmerspann, welche dieser am 12. Juni 1753 ablieferte. Er zählte acht Trauungen von Mistlauer Einwohnern zwischen 1708 und 1753 auf; an Beerdigungen waren es 21. Bei dieser Gelegenheit ist zu erfahren, dass Großallmerspann bis 1728 keinen eigenen Friedhof hatte; nachdem die katholischen Untertanen von Groß-Allmerspann nicht mehr auf dem evangelischen Friedhof in Lendsiedel beigesetzt werden sollten, waren sie bis dahin in Steinbach bei Schwäbisch Hall beigesetzt worden - immerhin eine Entfernung von rund $20 \mathrm{~km}$.

\section{Auf dem Weg zu einem Vergleich}

Am 9. November 1753 schrieb die bischöfliche Kanzlei von Würzburg dann an das Ritterstift Comburg, es sei zweifelhaft, ob Comburg das Jus Parochiale in Mistlau beanspruchen könne. Man möge doch einen gütlichen Ausgleich suchen. Bevor es dazu kam, wurde im Dezember 1753 bei den Bergers wieder ein Mädchen geboren. Auch auf Kirchberger Seite hatte inzwischen der Widerstand abgenommen. Diesmal waren es nur mit Stecken bewaffnete Bürger und keine Soldaten, die Notar Kleiner und den katholischen Pfarrer Stumpf daran hindern wollten, das Bergersche Haus zu betreten. Der Kirchberger Kammer-Sekretär verlangte zwar wieder einen Revers von Notar Kleiner, nachdem dieser verweigert wurde, verhinderte er aber nicht, dass Notar und Pfarrer ins Haus der Bergers gingen und der Pfarrer dort das Kind taufte. 


\section{Die Friedenskonferenz in Gaggstatt}

Allmählich war offenbar auf beiden Seiten die Einsicht gereift, dass bei diesem Streit für beide Seiten nichts zu gewinnen war. Man vereinbarte deshalb eine Art Friedenskonferenz ${ }^{16}$. Sie fand vom 20. bis 22. Februar 1754 in Gaggstatt statt. Von beiden Seiten waren je zwei Räte entsandt worden. Einer der beiden Kirchberger Gesandten war der Geheime Rat Heinrich Gottlieb Ephraim Fischer von Ingelfingen. Er war Vertreter des Seniorats der Hohenlohe-Neuensteinischen Linie. Inzwischen war die Angelegenheit also auf Hohenloher Seite auf die Ebene des Seniorats gehoben worden ${ }^{17}$. Den zweiten Abgeordneten auf Hohenloher Seite hatte Kirchberg entsandt; es war der Kanzlei-Rat Johann Ludwig Desiderius Ulmer. Die Comburger Abgeordneten waren der Ellwanger Dechant und Syndicus F. Hardt (also auch hier hatte man einen externen hochrangigen Verhandler hinzugezogen) und der uns inzwischen wohlbekannte Kommissarius und Kanzlei-Assessor A. O. Kleiner. Außerdem hatten sie noch einen Sekretär und Protokollanten mitgebracht, den Ellwanger Lic. jur. Baumann, des verstorbenen dasigen Herrn Vice-Canzler Baumanns Sohn, Schwager des Syndicus Hardt ${ }^{18}$.

Der äußere Ablauf der Gaggstadter Friedenskonferenz spielte sich nach dem Bericht der Hohenloher Deputierten so $\mathrm{ab}^{19}$ :

Am Montagabend, 18. Februar, traf Geheimrat Fischer von Ingelfingen kommend in Kirchberg ein. Den Dienstag verbrachte er zusammen mit Kanzlei-Rat Ulmer in der Kirchberger Kanzlei; sie sichteten Dokumente und bereiteten die Konferenz vor. Abends um 7 Uhr erhielten sie durch zwei Boten Nachricht aus Gaggstadt, dass die Comburger Delegation dort eingetroffen sei. Am nächsten Morgen fuhren sie in einer mit 6 Kutschen-Pferden bespanneten herrschaftlichen Chaise zur bestimmten Zeit nach Gaggstadt, langten dort um 9 Uhr an und bezogen ihr Quartier im Pfarrhaus. Sie ließen durch einen livrierten Bedienten die comburgische Delegation ihre Ankunft mitteilen. Diese ließ ihrerseits nachdem sie uns bey ihrem Quartier, so sie in dem Comburg. Wirth-Hauß genommen, vorbey fahren sehen, durch ihren Comburg. Schultheißen ein höfliches Bewillkommens-Compliment vermelden, und wegen der Zeit und Orts des Zusammentritts, sich bey uns erkundigen, worauf wir sowohl durch ihren abgeschickten, als meinen des geheimden

16 Quelle, soweit nicht anders angegeben, StAL B 375 L, Bü. 647.

17 Die Hohenloher Fürstentümer waren untereinander durch eine Senioratsverfassung verbunden. Jeweils der älteste Fürst vertrat die Hohenloher nach außen. Nachdem die Linie der Waldenburger zum katholischen Glauben konvertiert war, war das Seniorat geteilt worden. Die evangelischen Hohenloher hatten sich als die neuensteinische Linie organisiert. Senior war zur Zeit der Gaggstatter Konferenz der Fürst von Hohenlohe-Weikersheim.

18 Eine interessante Konstellation. Es ist ja anzunehmen, dass ein Vize-Kanzler in Kirchberg evangelisch war. Sein Sohn war jetzt in katholischen Diensten und verheiratet mit Schwester des Ellwanger Syndicus. Darf man annehmen, dass er die Konfession gewechselt hat?

19 HZA LA 35 Bü 677. 
Raths Bedienten, ein höfliches behöriges Gegen-Compliment machen, und zur Zusammenkunft das dießseitige Wirthshauß, wohin wir uns sofort begeben würden, benennen, und sie zugleich zu dem allda nach der Conferential-Handlung mit uns einzunehmenden Mittag-Essen invitiren.

Die Comburger waren mit Mietpferden angereist und hatten diese sofort nach ihrem Eintreffen zurückgeschickt. Die Kirchberger Delegation hatte deshalb - auch wegen des weiten und üblen Weegs von einem Wirthshauß zum anderen, der Comburger Delegation die herrschaftl. Chaise offeriren lassen, welches allselbige, nebst einem wiederum vermeldeten convenablen Compliment mit bezeigtem vielen Dank acceptirt hat.

Die Kirchberger Delegation fuhr also zum Kirchberger Wirtshaus und schickte die Kutsche weiter zum Comburgischen Wirtshaus. Als die Comburger ankamen, wurden sie bey dem Aussteigen von dem H.ren Canzley Secretarii Cunradt, von uns beyden aber heraußen vor der Thür empfangen, und in die unten an dem Eingang des Haußes befindl. wegen des noch nicht völlig zugerichteten oberen Stockwerks solches neu erbauten Wirthshaußes zu der Conferens genommenen großen Gast-Stube geführt. Dort hatte der herrschaftliche Laquay Albrecht schon alles gut vorbereitet: eine viereckige Tafel war aufgebaut, mit einem herrschaftlichen Tuch (Teppich) bedeckt und mit sechs Sesseln umstellt. Auch was das Mittagessen betrifft, hatte sich das Haus Kirchberg nicht lumpen lassen: Schon früh am Morgen hatte es einen Koch mit einem Küchenwagen nach Gaggstadt geschickt, der die Tafel mit Victualien reich und überflüßig (wohl als ,überfließend zu verstehen) versehen hatte.

Vor dem Mittagessen musste aber noch gearbeitet werden. Natürlich war die Sitzordnung in einem solchen Fall von Bedeutung - als Gäste erhielten die Comburger Deputierten die Plätze zur rechten Seite, während die Kirchberger als Gastgeber die Plätze zur linken Hand am Tisch einnehmen. Darauf folgte die erste Sitzungsrunde mit einer weitläuftigen, jedoch mit beyderseitigem vollkommen amicablen Bezeigen geschehener Diskussion. Dann - es war inzwischen 13 Uhr geworden - schritt man zum Mittagessen.

Wann die Diskussion danach fortgesetzt wurde, ist dem Bericht nicht zu entnehmen, wohl aber, wie lange man tagte: Und sind wir des nachts gegen 11 Uhr, nachdem man erst nach 9 Uhr zum Abend-Essen gekommen, beiderseits vergnügt wiederum auseinander gegangen, da vorhero die Comburg. $\mathrm{HH}$. Abgeordneten wieder in ihr Quartier in dem Comburg. Wirtshauß, wir aber in das Pfarr-Hauß, in der Herrschaftl. Chaise zurückgefahren. Vorher hatte man noch den Sitzungsbeginn am nächsten Morgen vereinbart und die Comburger Delegation hatte die Kirchberger zum Mittagessen am folgenden Tag eingeladen - und sich dazu den Herrschaftl. Koch Leicht zur Zubereitung des Essens höflich ausgebeten.

Die nächste Runde begann dann am folgenden Tag morgens um 9 Uhr im Comburgischen Quartier, nachdem die Kirchberger sich vorher schon intern 
beraten hatten. Bei der Begrüßung wurde wieder streng auf Rangfolge geachtet: Lic. jur Baumann ging den Kirchberger Delegierten bis an die Chaise entgegen, Syndicus Hardt und Kommissar Kleiner folgten einige Schritte zurück. Die Sitzung dauerte - mit Unterbrechung durch das Mittagessen wieder bis nachts um 11 Uhr. Große Mühe bereitete die Verfertigung eines Protokolls. Man vereinbarte, am nächsten Morgen wieder um 9 Uhr zur Unterzeichnung des Protokolls zusammen zu kommen, und zwar im Pfarrhaus. Der oder die Protokollanten (auf Kirchberger Seite ist keiner erwähnt - war das der oben erwähnte Kanzlei-Sekretär Cunradt?) mussten wohl am Abend oder vor dem Frühstück noch zwei Reinschriften verfertigen.

An nächstem Morgen wurde zunächst gefrühstückt; für die Comburger Delegation gab es Fastenspeise (es war Freitag). Danach wurden die beiden Exemplare des Protokolls auf Übereinstimmung überprüft und Abweichungen korrigiert. Dann gab es noch eine Auseinandersetzung wegen der Platzierung der Unterschriften. Die Kirchberger wollten auf der rechten Seite unterschreiben, den Comburgern wurde die linke Seite zugewiesen. Damit waren diese nicht einverstanden; sie schlugen vor - da ja zwei Exemplare vorhanden waren die Seiten jeweils zu wechseln. Die Kirchberger konterten, indem sie Kopien früherer Verträge hervorzogen, die sie mitgebracht hatten, auf welchen die Kirchberger Unterschriften auf der rechten, die Comburgischen auf der linken Seite zu sehen waren. Wodurch sich dann die jenseitige Deputati ohne weitere Widerstände eines besseren belehren ließen, und begriffen, und ihr Unterschrift ganz willig zur linken Hand gesezt haben.

Nun konnten also die beiden Dokumente unterschrieben und gesiegelt werden.

Danach reisten die Comburger Deputierten ab. Die Kirchberger Deputierten aber sind im übrigen, nachdem wir nach der vorher erfolgten Abreise derer Comburg. HH. Deputatorum, noch das Mittag-Essen eingenommen, und das Erforderliche mit dem H. Pfarrer abgeredet, von Gaggstadt wieder abgereist, und gestern Abends zwischen 4 und 5 Uhr Gottlob! glückl. hier wider zurück angelanget.

\section{Inhaltliche Diskussion und Protokoll}

Was war nun besprochen und verabredet worden? Zunächst einmal legten die Hohenloher Deputierten wohlformulierte Vollmachten vor. Die Comburger Seite zeigte sich überrascht - sie war davon ausgegangen, dass ihre vorherige schriftliche Anmeldung bei der Kirchberger Kanzlei genüge. Die Hohenloher meinten schließlich, der Mangel ließe sich notfalls im Rahmen der Ratifikation eines Abkommens heilen.

Natürlich hatten beide Seiten dann ausführlich ihre Standpunkte dargelegt. Einen 
Vergleich zur Güte machte dann vor dem Mittagessen im privaten Gespräch mit Geheimrat Fischer der Dechant Hardt für die Comburger Seite. Er schlug vor, dass der Groß-Allmerspanner Pfarrer weiterhin parochiale Akte in Mistlau durchführen dürfe, sofern er dafür jeweils eine Genehmigung vom Gaggstatter Pfarrer einholte. Auch sollten die Stolae, die Gebühren für einen solchen Akt an den Gaggstatter Pfarrer bezahlt werden. Auf ein solches Verfahren habe man sich z.B. auch in einem vergleichbaren Fall (wenn auch mit umgekehrten Vorzeichen) mit dem evangelischen Haus Berlichingen in Rechenberg geeinigt, das auf Ellwanger Gebiet liege. Die Hohenloher Seite war prinzipiell einer solchen Lösung nicht abgeneigt, erhob aber zusätzliche Forderungen: So meinte sie, das beste wäre doch, wann dem jezigen noch übrig seyenden Röm. Cath. Unterthan Berger zu Mistlau, von seiner Herrschaft erlaubt würde, nach seinem Wunsche anderwärts hinzuziehen ${ }^{20}$. Mindestens aber sollte von Comburger Seite die Versicherung gegeben werden, ermeltem Berger in einer gewissen Zeit jenseits eine anderweite Unterkunft zu verschaffen.

Außerdem verlangte sie, dass der Groß-Allmerspanner Pfarrer jedesmal den berühmten Revers de non praejudicando ausstellen und die Stolae nicht nur an den Pfarrer, sondern auch an den Organisten und den Mesner bezahlt werden müssten. Ein wichtiger Punkt war schließlich auch die Frage einer Entschädigung und Ehren-Erklärung für den Pfarrer Schäfer. Zum letzten Punkt meinte Dechant Hardt, es wäre doch das einfachste, wenn Pfarrer Schäfer dem Dechanten der Comburg einen Besuch abstatten würde - er werde diesmal sicher freundlicher empfangen als beim ersten Mal. Was eine finanzielle Entschädigung an Pfarrer Schäfer anbetrifft, zeigte sich Comburg hartleibig - und einen Termin für einen Abzug der Familie Berger aus Mistlau zu nennen, kam für Comburg natürlich überhaupt nicht in Frage. Um zu einem Ende zu kommen, beschloss man, die Punkte, auf die man sich einigen konnte, in einem Protokoll niederzulegen ${ }^{21}$. Das Ergebnis lässt sich so zusammenfassen:

Auf Comburgs Antrag: gegen die Entrichtung von Stolae an den Gaggstatter Pfarrer wird zugelassen, dass an in Mistlau befindlichen Personen katholische Parochial-Akte durchgeführt werden äußerte Hohenlohe wegen der grundsätzlich anderen Rechtsauffassung Bedenken. Um aber seine nachbarliche Gesinnung an den Tag zu legen, konnte die hohenlohische Delegation zustimmen, dass künftig an Bergers Familie und Wägeleins Witwe pfarramtliche Handlungen durch katholische Geistliche durchgeführt würden, wenn für die vergangenen Parochial-Akte die Stolae an Pfarrer, Schulmeister und Mesner bezahlt würden und dem Pfarrer von Gaggstatt Schadenersatz geleistet würde für erlittene Unbill, und wenn diese pfarramtlichen Handlungen 1. bei der Pfarrei Gaggstatt ge-

20 Wie aus dem Bericht zu entnehmen ist, gab es damals außer der Familie Berger nur noch des Schmidts Wägelin Wittib als katholische Einwohnerin in Mistlau. Im Haus Berger gab es die letzte Geburt und Taufe am 30. Januar 1756; im Jahr 1762 hat dann Michael Berger seinen Hof an einen Evangelischen verkauft: Berger (wie Anm. 1), S. 44.

21 StAL B 375 L Bü 647. 
hörig angezeigt würden, 2. die herkömmlichen Stolae gezahlt würden, 3. ein Revers de non praejudicando ausgestellt würde, die Akte in der Stille stattfinden und dem evangelischen Pfarrer gleiches Recht in Groß-Allmerspann eingeräumt würde. In Notfällen dürfe die Anzeige beim Pfarrer in Gaggstatt auch nachträglich geschehen.

Die comburgische Seite hielt demgegenüber fest: Ihrer Ansicht nach sei der rechtliche Vorbehalt schon genügend festgestellt worden (sc. also muss nicht jedes Mal ein neuer Revers ausgestellt werden). Der Beschränkung auf die Fam. Berger und die Witwe Wägelin könne nur unter dem Vorbehalt der Relation und Ratification (Bericht an die Herrschaft und Ratifizierung) zugestimmt werden. Man hoffe, dass im Rahmen der Ratifizierung zwischen den hohen Principalschaften die Frage der Entschädigung für die vergangenen Parochial-Akte und die Entschädigung für Pfarrer Schäfer geklärt werden könne bzw. man diese Frage im Blick auf ein zukünftiges gutnachbarliches Verhältnis auf sich beruhen lasse. Das Gegenrecht eines evangelischen Pfarrers in Groß-Allmerspann sei kein Problem unter der Voraussetzung, dass das Recht des katholischen Pfarrers in Mistlau über die Familie Berger hinaus ausgedehnt werde.

Die Hohenloher Seite antwortete: Man werde sich dafür einsetzen, dass die von Comburg gewünschte Ausweitung der Zulassung von Parochial-Akten auf andere sich in Mistlau befindliche katholische Personen im Rahmen der Ratifikation von der Hohenloher Principalschaft zugelassen werden. Voraussetzung sei aber in jedem Fall die Anzeige beim Pfarrer in Gaggstatt, die Entrichtung der Stolae an Pfarrer, Schulmeister und Mesner in Gaggstatt sowie die Ausstellung eines Reverses. Man hoffe außerdem weiterhin auf eine Entschädigung für Pfarrer Schäfer und Aufhebung und Rückgabe des von ihm in Würzburg ausgestellten Reverses, und man erinnerte daran, dass man weiterhin davon ausgehe, dass der evangelische Untertan Grombach in Groß-Allmerspann wie bisher von Lendsiedel aus pfarramtlich versorgt werden dürfe. Alles Weitere stellten die Delegationen der Ratifizierung durch die hohen Principalschaften anheim.

Wie man sieht: Man war sich im Grundsatz einig, überließ aber die Detail- und vor allem die finanziellen Fragen weiteren Verhandlungen im Rahmen der Ratifizierung. Der Quellenlage nach ist es zu einer solchen Ratifizierung nicht gekommen. Für die Hohenloher Seite dürfte eine solche Ratifizierung kein Problem gewesen sein - das Seniorat äußerte sich sehr zufrieden zum Ergebnis der Konferenz. Comburg scheint mit diesem Kompromiss nicht zufrieden gewesen zu sein. Würzburg antwortete jedenfalls an Comburg am 25. Januar 1756 auf eine (nicht mehr erhaltene) Anfrage von Comburg: Man möge doch bitte am gegenwärtigen Zustand nicht rütteln. In der Praxis dürfte man sich wohl stillschweigend an die grundsätzliche Einigung in der Gaggstatter Konferenz (Anzeige und Bezahlung der Stolae an den Gaggstatter Pfarrer) gehalten haben. Praktische Bedeutung hatte das ganze so gut wie keine mehr, nachdem es nur noch zwei Taufen im Haus Berger gab (20. April 1755 und 30. Januar 1756) und Michael Berger seinen Hof im Jahr 1762 an einen Evangelischen verkauft hatte. 


\section{Nachspiel: Wer soll das bezahlen?}

Spätestens mit der Vertretung des Hauses Hohenlohe durch Geheimrat Fischer bei der Gaggstatter Friedenskonferenz war der ganze Streit zu einer Sache der gesamten Linie Hohenlohe-Neuenstein geworden. Konsequenterweise fragte Hohenlohe-Kirchberg deshalb auch kurz nach der Gaggstatter Konferenz beim Seniorat nach, wie es mit der Übernahme der Kosten des Streites durch die Seniorats-Kasse stünde ${ }^{22}$. Die Seniorats-Regierung (damals in Weikersheim) machte Kirchberg Hoffnung auf Übernahme der Kosten und verlangte eine Kosten-Übersicht. Diese übersandte Kirchberg am 22. Juli 1754. Darin sind alle Kosten vom 2. Mai 1748 an aufgeführt. Insgesamt belaufen sich die aufgeführten Kosten auf $190 \mathrm{fl}, 1 \mathrm{x}$ und 2 Pfennige $^{23}$.

Die Weikersheimer Regierung ließ das Ersuchen der Kirchberger mit den gesamten Akten der Konferenz unter den Agnaten der Neuensteinischen Linie kursieren. Danach passierte - nichts. Erst drei Jahre (!) später, am 5. August 1757, fragte Kirchberg in Öhringen nach, was denn nun aus seinem Ersuchen geworden sei und schrieb: Da nun aber anjezo biß in das 3te Jahr hievon nicht das Geringste weiter zu vernehmen gewesen; so finden Wir uns veranlaßet, bey Eurer hochgräfl. Öhring. Wohllöbl. Seniorats-Regierungs-Canzley die Sache, mittelst die $\beta$, in gefällige Anerinnerung zu bringen, und von dem hierinnen etwa erfolgten hochherrschaftl. gemeinsamen gndgsten. Entschluß Uns die nähere Nachricht ergebenstens zu erbitten.

Doch auch diese Erinnerung scheint nicht von Erfolg gewesen zu sein. Erst fünf Jahre später, am 5. November 1762, konnte sich Kirchberg über die Nachricht von der Bewilligung des Geldes bedanken und um eine Anweisung an die Seniorats-Kasse in Öhringen bitten. Es scheint, dass Kirchberg diesmal ein Druckmittel in der Hand hatte - es war eine Umlage von $2000 \mathrm{fl}$ für die Auslagen aus der Seniorats-Kasse fällig. Damit war der Taufstreit in Mistlau auch geldmäßig endgültig abgeschlossen.

\section{Wer hatte Recht?}

Im Nachhinein fragt sich vielleicht der Leser, wer denn nun eigentlich Recht hatte. Deshalb soll zum juristischen Aspekt hier noch etwas gesagt werden. Auf beiden Seiten hatten die Juristen viel zu tun; entsprechend lang sind die Schriftsätze. Sie konzentrieren sich auf drei Fragenkreise:

22 Auch das Folgende wieder nach HZA LA 35 Bü 677.

23 Lt. Wikipedia besaß ein Gulden um 1700 eine Kaufkraft von 40 bis $50 €$. Nimmt man den höheren Wert, hätte das ganze Abenteuer die Kirchberger Grafschaft rund $10.000 €$ gekostet (die Arbeitsstunden der Kanzleien und Soldaten nicht einberechnet). Allerdings sind solche Gleichsetzungen früherer und heutiger Kaufkraft immer problematisch. 
1. Wer hat das Jus territoriale - also: wer hat die Territorial-Gewalt in Mistlau?

2. Wer hat das Jus Episcopale (die bischöfliche Gewalt) in Mistlau?

3. Wer hat das Jus Parochiale in Mistlau, wem stehen also die pfarrherrlichen Rechte in Mistlau zu?

Zur ersten Frage. Für uns ist es heute selbstverständlich, dass die Staatsgewalt in einem Gebiet nur bei einem einheitlichen Staat liegen kann. Dies ist aber ein neuzeitliches Verständnis. Das Mittelalter kommt her vom germanischen Gefolgs- und Lehensrecht. Demnach gehörte ein Untertan einem Herrscher zu, nicht einem Staatsgebiet. Comburg hatte im Mittelalter in Mistlau Güter teils geschenkt erhalten, teils erworben; die auf diesen Gütern Sitzenden wurden damit zu Comburger Untertanen. Gestritten wurde zwischen Kirchberg und Comburg um die Territorial-Gewalt. Diese umfasste im Wesentlichen die hohe Gerichtsbarkeit - also die Gerichtsbarkeit in den Fällen, in denen Strafen an Leib und Leben in Frage kamen. Zur Gerichtsbarkeit gehörte auch die Polizeigewalt - also das Recht, Untersuchungen und Festnahmen durchzuführen. Unbestritten zwischen Kirchberg und Comburg war, dass diese Gerichtsbarkeit im Hause dem jeweiligen Herren zustand, also den Comburgern über ihre und den Kirchbergern über ihre Untertanen. Gestritten wurde um die Gerichtsbarkeit auf „Gassen und Straßen“. Diese beanspruchte Kirchberg für sich und beruft sich dabei darauf, dass der Kaiser den Hohenlohern das Amt Kirchberg zum Lehen gegeben habe. Des Weiteren werden Gewohnheitsrecht und Präzedenzfälle ins Feld geführt. Comburg kann ebenfalls Präzedenzfälle und ein Urteil des Reichskammergerichts ins Feld führen. Über diese, die weltliche Seite des Streits und seinen Ausgang soll an anderer Stelle berichtet werden. Hier geht es um die kirchliche Gewalt, die mit der weltlichen eng verknüpft war.

Und damit zur zweiten Frage: Wem stehen die bischöflichen Rechte in Mistlau zu? Dass Territorial- und Kirchenherrschaft in einer Hand zu liegen haben, wird von beiden Seiten immer wieder betont. In den juristischen Schriftsätzen, welche ausgetauscht wurden, berufen sich beide Parteien ausgiebig und sich immer wiederholend auf den Westfälischen Frieden von 1648. Dort war der berühmte Grundsatz aufgestellt worden: cuius regio, eius religio - wessen die Herrschaft, dessen die Religion. Als Basisjahr war das Jahr 1624 bestimmt worden - die damals evangelischen Gebiete waren als evangelisch anzuerkennen ebenso wie die katholischen als katholisch. Von dorther war es eigentlich ganz klar, dass die bischöfliche Gewalt über Mistlau den Kirchbergern zustand.

Comburg argumentiert demgegenüber mit einer gewagten juristischen Konstruktion so: Im Westfälischen Frieden sei die katholische Episcopalgewalt über die protestantischen Gebiete nicht aufgehoben, sondern nur auf Zeit suspendiert worden, nämlich bis zur Wiedervereinigung der Kirchen (die sich Comburg natürlich unter dem Dach der katholischen Kirche vorstellt). Werden nun katholische Christen in evangelischen Gebieten angesiedelt, so lebe für diese Untertanen die katholische Episcopalgewalt wieder auf (reviviscentia juris episcopalis). 
Ob diese Konstruktion eine Erfindung der Comburger war oder damals auch anderswo vertreten wurde, habe ich noch nicht herausfinden können. Die Hohenloher halten dem entgegen, dass sich für diese Auslegung im Text der Friedensverträge keinerlei Anhaltspunkte finden und dass es auf diese Weise möglich wäre, durch Unterwanderung mit katholischen Untertanen wegen der Einheit von Jus Episcopale und Territoriale auch die territoriale Landkarte in Deutschland zu verändern.

Bleibt der dritte Punkt, die Parochial-Herrschaft. Dazu ist zum ersten zu sagen, dass dieser Punkt nicht so trivial ist, wie er uns heute erscheint. Pfarrer waren damals wichtige Amtsträger. Wie oben zu sehen, hatten sie nicht nur Hilfsdienste im Rahmen der Verwaltung zu erfüllen (,Seelenregister"). Das ganze Schulwesen und Sozialwesen (Armenfürsorge) lag in der Hand der pfarrherrlichen bzw. kirchlichen Verwaltung; selbst Totengräber und Hebamme wurden von der Pfarrverwaltung angestellt und bezahlt. Umgekehrt waren die Einnahmen aus Casualien ein nicht geringer Teil des Einkommens der Pfarrer, Schulmeister und Mesner. Vom Schulmeister in Lendsiedel gibt es eine Aufstellung, was ihm durch die Rekatholisierung in Groß-Allmerspann innerhalb von 11 Jahren an Einnahmen verloren gegangen sei. Er führte aus ${ }^{24}$ :

Was jetztmahliger Schuldiener zu Lendsidel ab A. 1687 incl. biß dato 1698 von Jahren zu Jahren, in dem eingepfarrten Filial Großen Allmerspann, an seiner rechtmäßigen Competenz ohne die accidentien zurück geblieben, und er kraft seiner Bestallung de Jure zu fordern.

\begin{tabular}{|l|c|c|c|}
\hline Anno & gingen $a b$ & & \\
\hline & Laib brod & Garben Frucht & Tröge $^{25}$ \\
\hline 1687 & 2 & 2 & 2 \\
\hline 1688 & 2 & 2 & 2 \\
\hline 1689 & 4 & 4 & 4 \\
\hline 1690 & 5 & 5 & 5 \\
\hline 1691 & 5 & 5 & 5 \\
\hline 1692 & 5 & 3 & 3 \\
\hline 1693 & 8 & 8 & 8 \\
\hline 1694 & 9 & 9 & 9 \\
\hline 1695 & 9 & 9 & 9 \\
\hline 1696 & 9 & 9 & 9 \\
\hline 1697 & 9 & 9 & 9 \\
\hline 1698 & & & 9 \\
\hline & $\mathbf{6 7}$ & $\mathbf{6 5}$ & 74 \\
\hline
\end{tabular}


$R S$ (Rescript $=$ Nachschrift). eod.(eodem $=$ gleichfalls) künftiger Erndt geliebts Gott, gehen 10 Garben und Brod ab.

Wann nun obige 67 Laib a 15 kr. einer gewehnet wird, thut

Eodem die 65 Garben a 8 kr. eine gewehent, thut $16 f l .45 \mathrm{kr}$

Ferner die 76 Trögel a 4 kr. gewehnet, thut

Und letztlich restiren von damahligen Schmid von den

letzten 5 Jahren hero 5. Michaelis

Summa

8 fl. $40 \mathrm{kr}$.

$5 \mathrm{fl} .4 \mathrm{kr}$

Lendsiedel, den 4. Maij 1698

Johann Caspar Melchior, Ludi mag. (Ludi magister = Schulmeister)“

Man vergleiche den Betrag von 30 fl 41 x mit den 190 fl, welchen der ganze Streit das Kirchberger Fürstenhaus (bzw. letztlich das Hohenloher Seniorat) gekostet hat. Für den Schulmeister mussten die 30 fl eine gehörige Einbuße sein.

Es ging also nicht nur um Ehrpusselei, wenn hier darum gestritten wurde, wer parochiale actus in der Gemeinde durchführen durfte. Wie oben schon dargestellt, war hier die Comburger Position angreifbar. Rechte über Pfarreien wurden schon im Mittelalter getrennt von Territorial- und Episkopal-Rechten vergeben, weiterverkauft und gekauft. Und es war nicht zweifelhaft, dass Hohenlohe die Rechte über die Pfarrei Gaggstatt inkl. Mistlau besaß. Insofern war es dann auch logisch, dass im Kompromiß von Gaggstatt 1754 diese Rechte insofern bestätigt wurden, als der katholische Pfarrer für seine Amtshandlungen die Genehmigung des evang. Pfarrers einzuholen hatte und an diesen Gebühren abzuführen waren.

Sei zum Schluss noch die Frage gestellt, was wohl Hohenlohe-Kirchberg bewogen hat, den Streit im Jahr 1747 so eskalieren zu lassen. Dazu zwei Gedanken: Zum einen war damals die Auseinandersetzung im Gange, die Jochen Vötsch als „Die Hohenloher Religionsstreitigkeiten“ beschrieben hat ${ }^{26}$. Auch da ging es um Rekatholisierungsbestrebungen, diesmal durch die Herrschaft Hohenlohe-Waldenburg, die 1667 zum Katholizismus übergetreten war. Dieser Konflikt erreichte in den Jahren von 1744 bis 1750 einen Höhepunkt. Auch hier ging der Konflikt bis hin zu einem Militäreinsatz (Besetzung Öhringens durch den Markgrafen von Brandenburg-Ansbach). Es ist denkbar, dass der Fürst von Hohenlohe-Kirchberg hier seinen Verwandten und Glaubensverwandten der Linie Hohenlohe-Neuenstein Schützenhilfe geben wollte - oder umgekehrt den Konflikt nutzen wollte, um seine eigene Position zu stärken.

Schließlich ist zu sehen, dass die Vermischung der drei Herrschaften in Mistlau eine unglückliche Konstruktion war, mit welcher beide Seiten (Crailsheim/Seckendorf spielte offenbar keine eigenständige Rolle) auf Dauer nicht zufrieden sein konnten. Die von Comburg betriebene Rekatholisierung dürfte auch dort 
mindestens ebenso sehr auf die weltliche Herrschaft im Gebiet Allmerspann-Mistlau ausgerichtet gewesen sein als auf die Ausbreitung des katholischen Glaubens - und beide Herrschaften darauf geschielt haben, irgendwann einmal die ganze Herrschaft an Mistlau übernehmen zu können. 